[4] W. Gordon, "Far-field approximations to the Kirchoff-Helmholtz representations of scattered fields," IEEE Trans. Antennas Propag., vol. 23, pp. 590-592, Jul. 1975.

[5] A. C. Ludwig, "Computation of radiation patterns involving numerical double integration," IEEE Trans. Antennas Propag., pp. 767-769, Nov. 1968.

[6] M. F. Cátedra, C. Delgado, S. Luceri, and F. S. de Adana, "Efficient procedure for computing fields created by current modes," Electron. Lett., vol. El-39, pp. 763-764, May 2003.

[7] M. F. Cátedra, C. Delgado, S. Luceri, O. Gutiérrez, and F. S. de Adana, "Physical optics analysis of multiple interactions in large scatters using current modes," IEEE Trans. Antennas Propag., vol. 54, pp. 985-994, Mar. 2006.

[8] L. Lozano, O. Gutierrez, and M. F. Cátedra, "Comparison between PO-GO, GO-PO and PO-PO applied to calculate the contribution of the double reflection between flat patches," presented at the IEEE AP-S Int. Symp. on Antennas Propag., Albuquerque, NM, Jul. 2006.

[9] G. Farin, Curves and Surfaces for Computer Aided Geometric Design: A Practical Guide. New York: Academic Press, 1988.

[10] O. Gutierrez, F. S. de Adana, F. Rivas, I. González, and M. F. Cátedra, "Method to interpolate the induced current with a low amount of sample points by means of Bézier surfaces," Electron. Lett., pp. 177-178, Jan. 2003.

[11] M. Abramowitch and I. A. Stegun, Handbook of Mathematical Functions With Formulas, Graphs and Mathematical Tables. New York: Wiley, 1964.

\section{On the Integral Identities Consisting of Two Spherical Bessel Functions}

\author{
Cheng-Wei Qiu, Le-Wei Li, Saïd Zouhdi, Tat-Soon Yeo, and \\ Qun $\mathrm{Wu}$
}

\begin{abstract}
When deriving dyadic Green's functions for the spherical structures with gyrotropic or bianisotropic materials, an integral whose integrand function consists of two spherical Bessel functions and a power function needs to be evaluated. Therefore, this paper revisits thoroughly the evaluation of the integral of $I_{l, l^{\prime}}\left(\kappa, \kappa^{\prime}\right)$. Starting from pointing out an error, it provides the correct solution to the integral in spherical coordinates in terms of distribution, in particular, step functions and delta functions. The formulation is further extended to a more generalized integral $H_{l, l^{\prime}}^{\lambda}\left(\kappa, \kappa^{\prime}\right)$; and it is newly found that the solution to the generalized integral varies differently in the cases of even and odd values of $l-l^{\prime}$. The mistakes that we found in the previous literature can also be proved easily by some of our intermediate solutions.
\end{abstract}

Index Terms-Bessel function, bianisotropic metamaterials, dyadic Green's functions (DGFs), electromagnetic field.

\section{INTRODUCTION}

The dyadic Green's functions (DGFs) [1], [2], as a mathematical kernel, relate directly the radiated electromagnetic fields and the source distribution, and this constitutes the basis of the integral equation methods (including the method of moments and the boundary element method). During the procedure of formulating the dyadic Green's functions for spherical structures with isotropic materials [3], chiral materials [4], and especially uniaxial anisotropic materials [5], the series of integral identities discussed in the present paper will not be encountered since the off-diagonal elements are zero in the material's parameter tensors. However, for the research of complex media and metamaterials (in which the bianisotropic effects will arise [6]), the problems of evaluation of the following integrals should be envisaged. This series of integrals is a key step in theoretically formulating the dyadic Green's functions for composite materials with bianisotropic properties (i.e., $\overline{\boldsymbol{\epsilon}}, \overline{\boldsymbol{\mu}}, \overline{\boldsymbol{\xi}}$, and $\overline{\boldsymbol{\zeta}}$, where $\overline{\boldsymbol{\xi}}$ and $\overline{\boldsymbol{\zeta}}$ are magnetoelectric pseudo-dyadics). Hence, due to the existence of off-diagonal elements, the Ohm-Rayleigh method can not be applied dierctly. When we use the vector eigenfunction expansion technique [1] for bianisotropic materials, the following integral consisting of spherical Bessel functions will be encountered:

$$
H_{l, l^{\prime}}^{(\lambda)}\left(\kappa, \kappa^{\prime}\right)=\int_{0}^{\infty} j_{l}(\kappa r) j_{l^{\prime}}\left(\kappa^{\prime} r\right) r^{-\lambda+1} d r
$$

where $j_{l}(\kappa r)$ denotes the spherical Bessel function of the first kind and $l, l^{\prime}$ and $\lambda$ are three integer numbers which may and may not be the same.

Manuscript received November 9, 2005; revised August 27, 2006

C.-W. Qiu, L.-W. Li and T.-S. Yeo are with Department of Electrical and Computer Engineering, National University of Singapore, Singapore 119260, Singapore.

S. Zouhdi is with Laboratoire de Génie Electrique de Paris, Ecole Supérieure D’Électricité, Gif-Sur-Yvette, Cedex, France.

Q. Wu is with Department of Electronics and Communications Engineering, Harbin Institute of Technology, Harbin 150001, China.

Digital Object Identifier 10.1109/TAP.2006.888467 
These integrals consisting of two Bessel functions are important and critical not only for engineers who calculate the electromagnetic fields and conduct antenna designs such as loop and aperture antennas, antenna radomes and radiation of antennas [7], [8] associated with the spherical layered structures [9], but also for physicists of atomic research who wish to characterize the periodic lattices of spheres [10], atomic interactions such as collisions, scatterings and quadrupole moments [11], [12]. In previous literature, due to the simplicity of the material parameters, these identities of integrals have not been envisaged. However, for the formulation of DGFs for bianisotropic media and for the antenna design involving bianisotropic metamaterials, these integrals have to be properly solved because Ohm-Rayleigh method can not be applied directly and all the exsiting integral identities are incapable to evaluate the current problem.

Hence, these integrals investigated in our paper are significantly important for the research of obtaining the dyadic Green's functions of composite materials with bianisotropic effects, characterizing the macroscopic performance of bianisotropic metamaterials, and studying of multipole moments of two- or three-body systems such as ${ }^{6} \mathrm{He}$ and ${ }^{6} \mathrm{Li}$. The $\lambda$ will be varied in order to cater for the different types of the particles considered. Finally, we gave the corrected solution to the integral $H_{l, l^{\prime}}^{(0)}\left(\kappa, \kappa^{\prime}\right)$ in [13] [the starting point of analyzing (1)], extended to a generalized problem, and made further explanation on [14]. In Section II, we made further derivation based on the results of Whelan. In the following section, proper solutions and generalization are presented.

Although Watson has discussed the integral $\int_{0}^{\infty}$ $J_{\mu}(a t) J_{\nu}(b t) t^{-\lambda} d t$, it actually bears the similar form as in (1). Its validity is only under the following convergence conditions:

$$
\begin{cases}\operatorname{Re}(\mu+\nu+1)>\operatorname{Re}(\lambda)>-1, & a \neq b \\ \operatorname{Re}(\mu+\nu+1)>\operatorname{Re}(\lambda)>0, & a=b .\end{cases}
$$

Moreover, most of the solutions in [14] were expressed in terms of hypergeometric functions, where the continuity (or discontinuity) has not been stated clearly and the Delta functions' presence (or absence) did not appear in all the derivations. This paper will not, therefore, follow directly Watson's results. Instead, we will revisit the integral in (1) when $\lambda=0$ and consider the Delta impulse and continuity. Based on that, (1) when $\lambda=1,2, \ldots$ can be calculated subsequently.

\section{IMPROPER SOLUTIONS IN [13]}

A general integral consisting two spherical Bessel functions and a power function as defined in (1) can be reduced to the following form:

$$
\begin{aligned}
H_{l, l^{\prime}}^{(\lambda)}\left(\kappa, \kappa^{\prime}\right)= & \int_{0}^{\infty} j_{l}(\kappa r) j_{l^{\prime}}\left(\kappa^{\prime} r\right) r^{-\lambda+1} d r \\
= & \sum_{p} \mathrm{~L}_{\lambda p}\left(\kappa, l, \kappa^{\prime}, l^{\prime}\right) \\
& \times \int_{0}^{\infty} r^{-\lambda+1} d r \int_{-1}^{1} j_{l}(h r) h^{-\lambda} P_{p}(\mu) d \mu \\
= & \sum_{p} \mathrm{~L}_{\lambda p}\left(\kappa, l, \kappa^{\prime}, l^{\prime}\right) \\
& \times \int_{-1}^{1} \frac{P_{p}(\mu)}{h^{2}} d \mu \int_{0}^{\infty} j_{\lambda}(t) t^{-\lambda+1} d t
\end{aligned}
$$

where the $P_{p}(\mu)$ denotes a Legendre function of the first kind and of the order $p, h=2 \kappa \kappa^{\prime}(\chi-\mu)$ and $\chi=\left(\kappa^{2}+\kappa^{\prime 2}\right) / 2 \kappa \kappa^{\prime}$, and $\mathrm{L}_{\lambda p}\left(\kappa, l, \kappa^{\prime}, l^{\prime}\right)$ is a function defined in [15].
In order to rewrite (2) more compactly, some identities will be introduced

$$
\begin{aligned}
\int_{0}^{\infty} j_{\lambda}(t) t^{-\lambda+1} d t & =\sqrt{\frac{\pi}{2}} \int_{0}^{\infty} \frac{J_{\lambda+\frac{1}{2}}(t)}{t^{\lambda-\frac{1}{2}}} d t \\
\int_{0}^{\infty} \frac{J_{\nu}(t)}{t^{\nu-\mu+1}} d t & =\frac{\Gamma(\mu / 2)}{2^{\nu-\mu+1} \Gamma(\nu-\mu / 2+1)}
\end{aligned}
$$

where $j_{l}(t)$ and $J_{\nu}(t)$ stand for the spherical Bessel function of order $l$ and cylindrical Bessel function of order $\nu$, respectively. After some manipulations, we can rewrite (2) as follows:

$$
\begin{aligned}
H_{l, l^{\prime}}^{(\lambda)}\left(\kappa, \kappa^{\prime}\right)= & \int_{0}^{\infty} j_{l}(\kappa r) j_{l^{\prime}}\left(\kappa^{\prime} r\right) r^{-\lambda+1} d r \\
= & \frac{1}{\kappa \kappa^{\prime}} \sum_{p} \mathrm{~L}_{\lambda p}\left(\kappa, l, \kappa^{\prime}, l^{\prime}\right) Q_{p}(\chi) \\
& \times \frac{\sqrt{\pi}}{2^{\lambda} \Gamma(\lambda+1 / 2)}
\end{aligned}
$$

where $Q_{p}(\chi)$ denotes the Legendre function of the second kind, and it satisfies the following form:

$$
Q_{p}(\chi)=\frac{1}{2} \int_{-1}^{1} \frac{P_{p}(\mu)}{\chi-\mu} d \mu
$$

When $\lambda=0$, we note that

$$
\mathrm{L}_{0 p}\left(\kappa, l, \kappa^{\prime}, l^{\prime}\right)=\frac{1}{2} \delta_{p l} \delta_{p l^{\prime}}
$$

which has been given by [15]. Substituting (6) into (2), we obtain

$$
H_{l, l^{\prime}}^{(0)}\left(\kappa, \kappa^{\prime}\right)= \begin{cases}0, & l \neq l^{\prime} ; \\ \frac{1}{2 \kappa \kappa^{\prime}} Q_{n}(\chi), & l=l^{\prime}=n\end{cases}
$$

which is yielded from Whelan's solution. Let us first introduce some classical identities as follows:

$$
\begin{aligned}
& \frac{\partial j_{n}(\kappa r)}{\partial \kappa} \\
& \quad=-\frac{j_{n}(\kappa r)}{2 \kappa}+\frac{r}{2}\left[j_{n-1}(\kappa r)-j_{n+1}(\kappa r)\right] \\
& \frac{\partial j_{n}(\kappa r)}{\partial r} \\
& \quad=\frac{\kappa}{2 n+1}\left[n j_{n-1}(\kappa r)-(n+1) j_{n+1}(\kappa r)\right] \\
& \frac{\partial\left[r j_{n}(\kappa r)\right]}{\partial r} \\
& \quad=\frac{\kappa r}{2 n+1}\left[(n+1) j_{n-1}(\kappa r)-n j_{n+1}(\kappa r)\right] \\
& j_{n}(\kappa r) \\
& \quad=\frac{\kappa r}{2 n+1}\left[j_{n-1}(\kappa r)+j_{n+1}(\kappa r)\right] \\
& \int_{0}^{\infty} j_{n}(\kappa r) j_{n}\left(\kappa^{\prime} r\right) r^{2} d r \\
& =\frac{\pi \delta\left(\kappa-\kappa^{\prime}\right)}{2 \kappa \kappa^{\prime}} .
\end{aligned}
$$


If we consider $H_{n, n}^{(1)}\left(\kappa, \kappa^{\prime}\right)$ and utilize (7) with (8), we have

$$
\begin{aligned}
H_{n, n}^{(1)}\left(\kappa, \kappa^{\prime}\right)= & \int_{0}^{\infty} j_{n}(\kappa r) j_{n}\left(\kappa^{\prime} r\right) d r \\
= & \frac{\kappa}{2 n+1} \cdot\left[\int_{0}^{\infty} j_{n-1}(\kappa r) j_{n}\left(\kappa^{\prime} r\right) r d r\right. \\
& \left.\quad+\int_{0}^{\infty} j_{n+1}(\kappa r) j_{n}\left(\kappa^{\prime} r\right) r d r\right] \\
= & \frac{\kappa}{2 n+1}\left[H_{n-1, n}^{(0)}\left(\kappa, \kappa^{\prime}\right)+H_{n+1, n}^{(0)}\left(\kappa, \kappa^{\prime}\right)\right] \\
= & 0
\end{aligned}
$$

which is obviously incorrect because $\int_{0}^{\infty} j_{n}(\kappa r) j_{n}\left(\kappa^{\prime} r\right) d r$ is not necessarily zero [14].

It can be observed that there is a limitation of rewriting (2) from integral form into the summation form [15], that is, $\lambda$ can not be zero. The mistake in previous work resides in applying (6) in (2). Since there is no other report discussing this problem and this problem is important in formulation of dyadic Green's functions for bianisotropic metamaterials, it thus motivates our objective of proposing a new method to evaluate $H_{l, l^{\prime}}^{\lambda}\left(\kappa, \kappa^{\prime}\right)$ from $\lambda=0$.

\section{Proper Solutions to The InTEgral of $H_{l, l^{\prime}}^{(0)}\left(\kappa, \kappa^{\prime}\right)$}

In this section, we show the significant differences of the solutions to the integral $H_{l, l^{\prime}}^{(0)}\left(\kappa, \kappa^{\prime}\right)$ when $l-l^{\prime}$ is even or odd. The results then fall into five categories:

1) $l=l^{\prime}=n$;

2) $l-1=l^{\prime}$

3) $l+1=l^{\prime}$;

4) Even $l-1-l^{\prime}$ but $l \pm 1 \neq l^{\prime}$ : a) $l-1>l^{\prime}$; b) $l+1<l^{\prime}$; and c) $l-1<l^{\prime}<l+1$; and

5) Odd $l-1-l^{\prime}$ where $\left(l \neq l^{\prime}\right)$.

Before the subsequent exact derivation, we consider the following identity, which will be employed in the near future:

$$
I_{l, l^{\prime}}\left(\kappa, \kappa^{\prime}\right)=\int_{0}^{\infty} j_{l}(\kappa r) j_{l^{\prime}}\left(\kappa^{\prime} r\right) r^{2} d r
$$

Although this integral does not converge into a finite value, it can be expressed in terms of distributions, in particular, step functions and Dirac delta functions. Starting from [14], we consider $I_{l, l^{\prime}}^{c}\left(\kappa, \kappa^{\prime}\right)$ with a convergence factor $c$ which can be sufficiently small so as to evaluate $I_{l, l^{\prime}}\left(\kappa, \kappa^{\prime}\right)$

$$
\begin{aligned}
I_{l, l^{\prime}}^{c}\left(\kappa, \kappa^{\prime}\right) & \int_{0}^{\infty} j_{l}(\kappa r) j_{l^{\prime}}\left(\kappa^{\prime} r\right) r^{2-c} d r \\
= & \frac{\pi}{2 \sqrt{\kappa \kappa^{\prime}}} \int_{0}^{\infty} J_{l+\frac{1}{2}}(\kappa r) J_{l^{\prime}+\frac{1}{2}}\left(\kappa^{\prime} r\right) r^{1-c} d r \\
= & \frac{2^{-c} \pi \kappa_{<}^{l^{\prime}}}{\kappa_{>}^{l^{\prime}+3-c}} \frac{\Gamma\left[\left(l+l^{\prime}+3-c\right) / 2\right]}{\Gamma\left(l^{\prime}+3 / 2\right) \Gamma\left[\left(l-l^{\prime}+c\right) / 2\right]} \\
& \times{ }_{2} F_{1}\left(\frac{l+l^{\prime}+3-c}{2}, \frac{l-l^{\prime}-c}{2}+1 ; l^{\prime}+\frac{3}{2} ; \frac{\kappa_{<}^{2}}{\kappa_{>}^{2}}\right)
\end{aligned}
$$

where $\kappa_{>}=\max \left(\kappa, \kappa^{\prime}\right)$ and $\kappa_{<}=\min \left(\kappa, \kappa^{\prime}\right)$.

$\kappa>\kappa^{\prime}>0$ is assumed to maintain the convergence and ${ }_{2} F_{1}$ is the hypergeometric function [16]. Using those important properties of $I_{l, l^{\prime}}^{c}\left(\kappa, \kappa^{\prime}\right)$ and hypergeometric functions formulated in Appendix, we can rewrite (10), for even $l-l^{\prime}$, given for $l \gtrless l^{\prime}$ as follows:

$$
\begin{array}{r}
I_{l, l^{\prime}}\left(\kappa, \kappa^{\prime}\right) \\
=\left\{\begin{array}{l}
g_{l, l^{\prime}}\left(\kappa, \kappa^{\prime}\right) U\left(\kappa-\kappa^{\prime}\right)+\frac{\pi}{2 \kappa \kappa^{\prime}}(-1)^{\left(l-l^{\prime}\right) / 2} \delta\left(\kappa-\kappa^{\prime}\right) \\
g_{l^{\prime}, l}\left(\kappa^{\prime}, \kappa\right) U\left(\kappa^{\prime}-\kappa\right)+\frac{\pi}{2 \kappa \kappa^{\prime}}(-1)^{\left(l^{\prime}-l\right) / 2} \delta\left(\kappa-\kappa^{\prime}\right)
\end{array}\right.
\end{array}
$$

where the function of $g_{l, l^{\prime}}\left(\kappa, \kappa^{\prime}\right)$ has also been given in Appendix so that $g_{l^{\prime}, l}\left(\kappa^{\prime}, \kappa\right)$ can be obtained straightforwardly by exchanging $l$ with $l^{\prime}$ and $\kappa$ with $\kappa^{\prime}$, and the function $U\left(\kappa-\kappa^{\prime}\right)$ denotes a unit step function defined by

$$
U\left(\kappa-\kappa^{\prime}\right)= \begin{cases}1, & \kappa>\kappa^{\prime} \\ 0, & \kappa<\kappa^{\prime}\end{cases}
$$

On the other hand, the form of the result of $I_{l, l^{\prime}}\left(\kappa, \kappa^{\prime}\right)$ for $l-l^{\prime}$ odd is given below

$$
I_{l, l^{\prime}}\left(\kappa, \kappa^{\prime}\right)=g_{l, l^{\prime}}\left(\kappa, \kappa^{\prime}\right) U\left(\kappa-\kappa^{\prime}\right)+g_{l^{\prime}, l}\left(\kappa^{\prime}, \kappa\right) U\left(\kappa^{\prime}-\kappa\right) .
$$

The factor of $(-1)^{\left(l-l^{\prime}\right) / 2}$ is due to the asymptotic assumptions for large arguments of Bessel functions [17] when $\kappa$ is very close to $\kappa^{\prime}$ and the detailed procedures of this near-region method were well presented by Qiu et al. [18]. In addition, the identity of (8d) will be widely used in the following derivations.

\section{A. Integral for $l=l^{\prime}=n$}

Substituting (8d) into (1), we have

$$
H_{n, n}^{(0)}\left(\kappa, \kappa^{\prime}\right)=\frac{\kappa}{2 n+1}\left[I_{n-1, n}\left(\kappa, \kappa^{\prime}\right)+I_{n+1, n}\left(\kappa, \kappa^{\prime}\right)\right] .
$$

After some manipulations, we arrive at

$$
\begin{aligned}
& H_{n, n}^{(0)}\left(\kappa, \kappa^{\prime}\right) \\
& \quad=\frac{\kappa}{2 n+1}\left[\left[g_{n-1, n}\left(\kappa, \kappa^{\prime}\right)+g_{n+1, n}\left(\kappa, \kappa^{\prime}\right)\right] \times U\left(\kappa-\kappa^{\prime}\right)\right. \\
& \left.\quad+\left[g_{n, n-1}\left(\kappa^{\prime}, \kappa\right)+g_{n, n+1}\left(\kappa^{\prime}, \kappa\right)\right] \times U\left(\kappa^{\prime}-\kappa\right)\right] .
\end{aligned}
$$

\section{B. Integral for $l-1=l^{\prime}$}

Substituting (8d) into (1), we have

$$
H_{l, l^{\prime}}^{(0)}\left(\kappa, \kappa^{\prime}\right)=\frac{\kappa}{2 l+1}\left[I_{l-1, l-1}\left(\kappa, \kappa^{\prime}\right)+I_{l+1, l-1}\left(\kappa, \kappa^{\prime}\right)\right] .
$$

Using the identities given in this section, we arrive at

$$
H_{l, l^{\prime}}^{(0)}\left(\kappa, \kappa^{\prime}\right)=\frac{\kappa}{2 l+1} g_{l+1, l-1}\left(\kappa, \kappa^{\prime}\right) U\left(\kappa-\kappa^{\prime}\right)
$$

In Fig. 1, one can see that the value of integral of $H_{4,3}^{(0)}(\kappa, 3)$ is decaying along $\kappa$. If $\kappa \leq \kappa^{\prime}$, it is zero which can be seen from (18).

It is also obvious that (7), which is the results in Whelan's work, is wrong since the value is not zero when $l \neq l^{\prime}$.

\section{Integral for $l+1=l^{\prime}$}

Substituting (8d) into (1), we have

$$
H_{l, l^{\prime}}^{(0)}\left(\kappa, \kappa^{\prime}\right)=\frac{\kappa}{2 l+1}\left[I_{l-1, l+1}\left(\kappa, \kappa^{\prime}\right)+I_{l+1, l+1}\left(\kappa, \kappa^{\prime}\right)\right]
$$




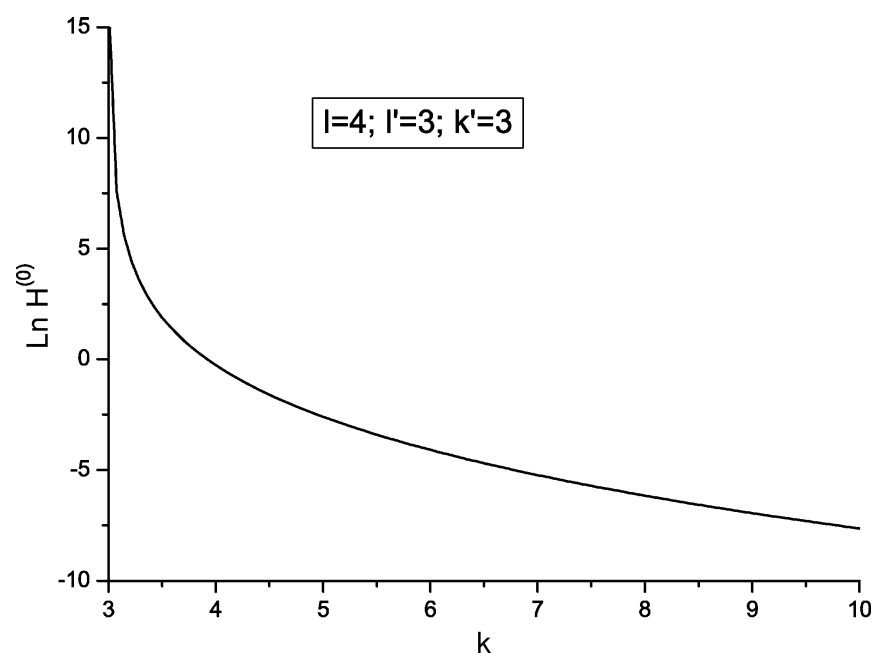

Fig. 1. An example of $H_{4,3}^{(0)}(\kappa, 3)$ versus $\kappa$.

Using the identities given in this section, we obtain

$$
H_{l, l^{\prime}}^{(0)}\left(\kappa, \kappa^{\prime}\right)=\frac{\kappa}{2 l+1} g_{l+1, l-1}\left(\kappa^{\prime}, \kappa\right) U\left(\kappa^{\prime}-\kappa\right)
$$

D. Integral for Even $l-1-l^{\prime}$ but $l \pm 1 \neq l^{\prime}$

Substituting (8d) into (1), we have

$$
H_{l, l^{\prime}}^{(0)}\left(\kappa, \kappa^{\prime}\right)=\frac{\kappa}{2 l+1}\left[I_{l-1, l^{\prime}}\left(\kappa, \kappa^{\prime}\right)+I_{l+1, l^{\prime}}\left(\kappa, \kappa^{\prime}\right)\right] .
$$

1) $l-1>l^{\prime}$ : Expanding (21) in this condition, we have

$$
\begin{aligned}
H_{l, l^{\prime}}^{(0)}\left(\kappa, \kappa^{\prime}\right)=\frac{\kappa}{2 l+1}[ & g_{l-1, l^{\prime}}\left(\kappa, \kappa^{\prime}\right) U\left(\kappa-\kappa^{\prime}\right) \\
& +(-1)^{\left(l-1-l^{\prime}\right) / 2} \delta\left(\kappa-\kappa^{\prime}\right) \\
& +g_{l+1, l^{\prime}}\left(\kappa, \kappa^{\prime}\right) U\left(\kappa-\kappa^{\prime}\right) \\
& \left.\quad(-1)^{\left(l+1-l^{\prime}\right) / 2} \delta\left(\kappa-\kappa^{\prime}\right)\right] \\
= & \frac{\kappa U\left(\kappa-\kappa^{\prime}\right)}{2 l+1} \\
& \times\left[g_{l-1, l^{\prime}}\left(\kappa, \kappa^{\prime}\right)+g_{l+1, l^{\prime}}\left(\kappa, \kappa^{\prime}\right)\right] .
\end{aligned}
$$

Note that the two parts of delta functions cancel each other.

2) $l+1<l^{\prime}$ : Expanding (21) under this condition, we obtain

$$
\begin{aligned}
H_{l, l^{\prime}}^{(0)}\left(\kappa, \kappa^{\prime}\right)= & \frac{\kappa}{2 l+1}\left[g_{l^{\prime}, l-1}\left(\kappa^{\prime}, \kappa\right) U\left(\kappa^{\prime}-\kappa\right)\right. \\
& +(-1)^{\left(l^{\prime}-l+1\right) / 2} \delta\left(\kappa-\kappa^{\prime}\right) \\
& +g_{l^{\prime}, l+1}\left(\kappa^{\prime}, \kappa\right) U\left(\kappa^{\prime}-\kappa\right) \\
& \left.+(-1)^{\left(l^{\prime}-l-1\right) / 2} \delta\left(\kappa-\kappa^{\prime}\right)\right] \\
= & \frac{\kappa U\left(\kappa^{\prime}-\kappa\right)}{2 l+1} \\
& \times\left[g_{l^{\prime}, l-1}\left(\kappa^{\prime}, \kappa\right)+g_{l^{\prime}, l+1}\left(\kappa^{\prime}, \kappa\right)\right] .
\end{aligned}
$$

3) $l-1<l^{\prime}<l+1$ : This category is actually the $l=l^{\prime}$ that has been discussed in Section III-A.

\section{E. Integral for Odd $l-1-l^{\prime}$}

Expanding (21) under this specific condition, we have

$$
\begin{aligned}
H_{l, l^{\prime}}^{(0)}\left(\kappa, \kappa^{\prime}\right)= & \frac{\kappa}{2 l+1}\left[\left(g_{l-1, l^{\prime}}\left(\kappa, \kappa^{\prime}\right)+g_{l+1, l^{\prime}}\left(\kappa, \kappa^{\prime}\right)\right) U\left(\kappa-\kappa^{\prime}\right)\right. \\
& \left.+\left(g_{l^{\prime}, l-1}\left(\kappa^{\prime}, \kappa\right)+g_{l^{\prime}, l+1}\left(\kappa^{\prime}, \kappa\right)\right) U\left(\kappa^{\prime}-\kappa\right)\right] .
\end{aligned}
$$

Now, all the situations of the integral $H_{l, l^{\prime}}^{(0)}\left(\kappa, \kappa^{\prime}\right)$ have been considered, and their respective solutions are obtained. The forms of $H_{l, l^{\prime}}^{(0)}\left(\kappa, \kappa^{\prime}\right)$ are obviously different from the expression in [13]. If $l=l^{\prime}=n$, according to the (A-6) and (12), we will arrive at

$$
I_{n, n}\left(\kappa, \kappa^{\prime}\right)=\frac{\pi}{2 \kappa \kappa^{\prime}} \delta\left(\kappa-\kappa^{\prime}\right)
$$

which agrees with that in (8e). This result in delta's form is also identical with the proof in Mathematica of Wolfram Research, Inc. Hence, the correctness of our present results can thus be verified once again. By using the new solution of this paper, we can treat $\int_{0}^{\infty} j_{l}(\kappa r) j_{l^{\prime}}\left(\kappa^{\prime} r\right) r^{n} d r$ with arbitrary values of $l, l^{\prime}, \kappa, \kappa^{\prime}$, and $n$.

$$
\text { IV. Generalization From } H_{l, l^{\prime}}^{(0)}\left(\kappa, \kappa^{\prime}\right) \text { To } H_{l, l^{\prime}}^{(\lambda)}\left(\kappa, \kappa^{\prime}\right)
$$

After obtaining $H_{l, l^{\prime}}^{(0)}\left(\kappa, \kappa^{\prime}\right)$ under different circumstances, we are now able to evaluate the $H_{l, l^{\prime}}^{(\lambda)}\left(\kappa, \kappa^{\prime}\right)$ in (1). By employing (8d), we can move a step further as follows:

$$
\begin{aligned}
H_{l, l^{\prime}}^{(\lambda+1)}\left(\kappa, \kappa^{\prime}\right) & =\int_{0}^{\infty} j_{l}(\kappa r) j_{l^{\prime}}\left(\kappa^{\prime} r\right) r^{-(\lambda+1)+1} d r \\
& =\int_{0}^{\infty} \frac{\kappa\left[j_{l-1}(\kappa r)+j_{l+1}(\kappa r)\right]}{2 l+1} j_{l^{\prime}}\left(\kappa^{\prime} r\right) r^{-\lambda+1} d r \\
& =\frac{\kappa}{2 l+1}\left[H_{l-1, l^{\prime}}^{(\lambda)}\left(\kappa, \kappa^{\prime}\right)+H_{l+1, l^{\prime}}^{(\lambda)}\left(\kappa, \kappa^{\prime}\right)\right]
\end{aligned}
$$

Therefore, once the solution of $H_{l, l^{\prime}}^{(0)}\left(\kappa, \kappa^{\prime}\right)$ as an initial value is given in Section II, we can evaluate subsequently (1) with an arbitrary positive non-zero $\lambda$, in an iterative procedure. The details are suppressed here due to the length restriction of the paper.

\section{CONCLUSION}

This paper presents a new evaluation of the integral $H_{l, l^{\prime}}^{(0)}\left(\kappa, \kappa^{\prime}\right)$ with the delta impulses extracted out and considers all the cases with respect to $l$ and $l^{\prime}$. Through evaluation of the integral $H_{l, l^{\prime}}^{(0)}\left(\kappa, \kappa^{\prime}\right)$, it is found that the integral $I_{0}\left(\kappa, l, \kappa^{\prime}, l^{\prime} ; 0\right)$ in [13] was incorrectly obtained, mainly due to the limitation of the value of $\lambda$. It is realized that there exists a limitation of $\lambda \geq 1$ in (2) and (3a). Although this condition was pointed out in [19], however, Whelan mis-adopted the solution of Seaton in [15] where $\lambda$ could be zero, when evaluating the integral. With the correct solutions of $H_{l, l^{\prime}}^{(0)}\left(\kappa, \kappa^{\prime}\right)$, we can obtain the integral $\int_{0}^{\infty} j_{l}(\kappa r) j_{l^{\prime}}\left(\kappa^{\prime} r\right) r^{n} d r$ when $n \leq 0$ using the procedures given in Section IV, which will be of great use in DGF formulation and atomic research as indicated in the introduction.

\section{APPENDIX}

In (11), letting $c$ approach $0^{+}$, we can obtain the expressions for $I_{l, l^{\prime}}\left(\kappa, \kappa^{\prime}\right)$

$$
\begin{aligned}
I_{l, l^{\prime}}\left(\kappa, \kappa^{\prime}\right)= & \frac{\pi \kappa_{<}^{l^{\prime}}}{\kappa_{>}^{l^{\prime}+3}} \frac{\Gamma\left[\left(l+l^{\prime}+3\right) / 2\right]}{\Gamma\left(l^{\prime}+3 / 2\right) \Gamma\left[\left(l-l^{\prime}\right) / 2\right]} \\
& \times{ }_{2} F_{1}\left(\frac{l+l^{\prime}+3}{2}, \frac{l-l^{\prime}}{2}+1 ; l^{\prime}+\frac{3}{2} ; \frac{\kappa_{<}^{2}}{\kappa_{>}^{2}}\right) .
\end{aligned}
$$

Note that the hypergeometric function ${ }_{2} F_{1}$ will encounter a complex infinity when $\kappa=\kappa^{\prime}$. That's why the delta function is included in final form of $I_{l, l^{\prime}}\left(\kappa, \kappa^{\prime}\right)$ even if $l \neq l^{\prime}$ as in (12). Subsequently, the expression for $g_{l, l^{\prime}}\left(\kappa, \kappa^{\prime}\right)$ can be formulated:

$$
g_{l, l^{\prime}}\left(\kappa, \kappa^{\prime}\right)=I_{l, l^{\prime}}\left(\kappa, \kappa^{\prime}\right), \quad \kappa \neq \kappa^{\prime}
$$


Based on some special properties of hypergoemtric as stated in [20], we further express the ${ }_{2} F_{1}$ in terms of Legendre polynomial and $\Gamma$ functions

$$
\begin{aligned}
{ }_{2} F_{1} & \left(\frac{l+l^{\prime}+3}{2}, \frac{l^{\prime}-l}{2}+1 ; l^{\prime}+\frac{3}{2} ; z^{2}\right) \\
= & \frac{2(-1)^{l^{\prime}} \Gamma\left(\frac{3}{2}+l^{\prime}\right) \Gamma(p) \Gamma(p+1)}{\sqrt{\pi}\left(1-z^{2}\right)^{p} \Gamma(q) \Gamma(q+1)} \times \frac{d^{l^{\prime}}}{d\left(z^{2}\right)^{l^{\prime}}} \\
& \times\left[{ }_{2} F_{1}\left(p+1, \frac{3}{2}-p ; \frac{3}{2} ; z^{2}\right)\left(1-z^{2}\right)^{q}\right]
\end{aligned}
$$

where

$$
\begin{gathered}
p=\frac{l-l^{\prime}+1}{2}, \quad q=\frac{l+l^{\prime}+1}{2}, \quad z=\frac{\kappa_{<}}{\kappa_{>}} \\
{ }_{2} F_{1}\left(p+1, \frac{3}{2}-p ; \frac{3}{2} ; z^{2}\right) \\
=-\frac{\Gamma\left[p+\frac{1}{2}\right] \Gamma[2 p-1] \Gamma[1-p]}{z \sqrt{\pi} \Gamma[2 p+1]} \times \frac{d}{d z} P_{2 p-1}(z) .
\end{gathered}
$$

Note that when $l=l^{\prime}=n$ (namely, $p=1 / 2$ ) we have

$$
\frac{d}{d z} P_{0}(z)=0
$$

Hence, we have

$$
g_{n, n}\left(\kappa, \kappa^{\prime}\right)=0
$$

which results in the conclusion of (25).

\section{ACKNOWLEDGMENT}

The authors are grateful to the SUMMA Foundation, Albuquerque, NM. C.-W. Qiu is grateful to the Joint Ph.D. Degree Program offered by the National University of Singapore, in Singapore, and Supélec, in Paris, France.

\section{REFERENCES}

[1] C. T. Tai, Dyadic Green's Functions in Electromagnetic Theory, 2nd ed. Piscataway, NJ: IEEE Press, 1994.

[2] J. A. Kong, Electromagnetic Wave Theory, 3rd ed. New York: Wiley, 1990.

[3] L. W. Li, P. S. Kooi, M. S. Leong, and T. S. Yeo, "Electromagnetic dyadic Green's function in spherically multilayered media," IEEE Trans. Microw. Theory Tech., vol. 42, no. 12, pt. A, pp. 2302-2310, Dec. 1994.

[4] _ "A general expression of dyadic Green's function in radially multilayered chiral media," IEEE Trans. Antennas Propag., vol. 43, no. 3, pp. 232-238, Mar. 1995.

[5] S. Liu, L.-W. Li, M.-S. Leong, and T.-S. Yeo, "Field representations in general rotationally uniaxial anisotropic media using spherical vector wave functions," Microw. Opt. Technol. Letters., vol. 25, no. 3, pp. 159-162, May 2000.

[6] W. Xu, L. W. Li, H. Y. Yao, T. S. Yeo, and Q. Wu, "Extraction of constitutive relation tensor parameters of SRR structures using transmission line theory," J. Electromag. Waves Appl., vol. 20, no. 1, pp. 13-25, Jan. 2006.

[7] L. W. Li, M. S. Leong, P. S. Kooi, T. S. Yeo, and Y. L. Qiu, "Radiation of an aperture antenna covered by a spherical shell chiral radome and fed by a circular waveguide," IEEE Trans. Antennas Propag., vol. 46, no. 5, pp. 664-671, May 1998.

[8] L.-W. Li, M.-S. Leong, and Y. Huang, "Elelctromagnetic radiation of antennas in the presense of an arbitrarily shaped dielectric object: Green dyadics and their applications," IEEE Trans. Antennas Propag., vol. 49, no. 1, pp. 84-90, Jan. 2001.

[9] I. V. Lindell, M. E. Ermutlu, and A. H. Shivola, "Electrostatic image theory of layered dielectric sphere," Proc. Inst. Elect. Eng. Microwaves, Antennas and Propagation, vol. 139, pt. H, pp. 186-192, Apr. 1992.
[10] N. A. Nicorovici, R. C. McPhedran, and K.-D. Bao, "Propagation of electromagnetic waves in periodic lattices of spheres: Green's function and lattice sums," Phys. Rev. E, vol. 51, no. 1, pp. 690-702, Jan. 1995.

[11] C. Leubner and E. M. Strohmaier, "Analysis of intensity effects in laser electron-beam backscattering in terms of generalised Bessel functions," J. Phys. A: Math. Gen, vol. 14, pp. 509-520, Feb. 1981.

[12] D. R. Lehman and W. C. Parke, "Shell structure of the $A=6$ ground states from three-body dynamics," Phys. Rev. C, vol. 28, no. 1, pp. 364-382, July 1983.

[13] C. T. Whelan, "On the Bethe approximation to the reactance matrix," J. Phys. B: At. Mol. Phys., vol. 19, no. 15, pp. 2343-2354, Aug. 1986.

[14] G. N. Watson, A Treatise on the Theory of Bessel Functions, 2nd ed. Cambridge, U.K.: Cambridge Univ. Press, 1966.

[15] M. J. Seaton, "The evaluation of partial wave integrals in the Born approximation," Proc. Phys. Soc., vol. 77, no. 1, pp. 184-191, Jan. 1961.

[16] H. M. Srivastava and R. G. Buschman, Theory and Applications of Convolution Integral Equations. Boston, MA: Kluwer Academic, 1992.

[17] R. E. Collin, Field Theory of Guided Waves, 2nd ed. Piscataway, NJ: IEEE Press, 1991.

[18] C.-W. Qiu, L.-W. Li, T.-S. Yeo, and S. Zouhdi, "Computational study of significant semi-infinite integrals in electromagnetic and atomic interactions," in Proc. 17th Int. Zurich Symp. Electromagnetic Compatibility, 2006, pp. 204-207.

[19] I. S. Gradshteyn and I. M. Ryzhik, Tables of Integrals, Series and Products. New York: Academic Press, 1965.

[20] Bateman Manuscript Project, Higher Transcendental Functions McGraw-Hill. New York, 1953, Based, in part, on notes left by Harry Bateman, and compiled by the staff of the Bateman Manuscript Project, Edited by Erdélyi, Vol 1. 